\title{
Some New Remarks about the Dynamics of an Automobile with Two Trailers
}

\author{
Camelia Petrişor \\ Department of Mathematics, "Politehnica” University Timişoara, Piaţa Victoriei, No. 2, 300006 Timişoara, Romania \\ Correspondence should be addressed to Camelia Petrişor; galeacamelia@yahoo.com
}

Received 14 March 2014; Revised 17 May 2014; Accepted 23 May 2014; Published 11 June 2014

Academic Editor: Kazutake Komori

Copyright (C) 2014 Camelia Petrişor. This is an open access article distributed under the Creative Commons Attribution License, which permits unrestricted use, distribution, and reproduction in any medium, provided the original work is properly cited.

The goal of our paper is to complete some results presented by Craioveanu et al. (1998) concerning the nonlinear stability of the equilibrium states of the car with two trailers' dynamics. In addition, the Lax formulation, numerical integration via Lie-Trotter, and Kahan's integrator for these dynamics are presented.

\section{Introduction}

The dynamics of a kinematic model of an automobile with two trailers were first described by Leonard (see [1]) as a chained form system. Later, the system was studied as a left invariant control system on a matrix Lie group in [2]. In the same paper, the Hamilton-Poisson structure of the system was presented together with some geometrical and dynamical properties. The goal of our paper is to complete some of these results.

The paper is structured as follows: the first part presents the Hamilton-Poisson structure of the systems from [2]. The Casimir functions corresponding to this structure are found. Beginning with these Casimirs, two new Hamilton-Poisson structures of the system are proposed. The second paragraph analyzes the nonlinear stability of the equilibrium states of the dynamics. Due to the existence of two Casimirs, we do not need to employ a function control to obtain the stability results, like in [2]. The Lax formulation of the system is the subject of the third paragraph. In the last part of the paper we discuss numerical integration of the dynamics via two methods. A numerical simulation for both results is also presented.

\section{The Geometrical Overview of the Problem}

The dynamics of the car with two trailers have been studied as a mechanical problem on a matrix Lie group. Following [2], the system that describes the dynamics is given by

$$
\begin{gathered}
\dot{x_{1}}=x_{2} x_{3}, \\
\dot{x_{2}}=-x_{1} x_{3}, \\
\dot{x_{3}}=-x_{1} x_{4}, \\
\dot{x_{4}}=-k x_{1}
\end{gathered}
$$

and may be realized as a Hamilton-Poisson system with the phase space $R^{4}$; the Poisson structure is given by the matrix

$$
\Pi=\left[\begin{array}{cccc}
0 & x_{3} & x_{4} & k \\
-x_{3} & 0 & 0 & 0 \\
-x_{4} & 0 & 0 & 0 \\
-k & 0 & 0 & 0
\end{array}\right]
$$

and the Hamiltonian $H\left(x_{1}, x_{2}, x_{3}, x_{4}\right)=(1 / 2)\left(x_{1}^{2}+x_{2}^{2}\right)$, and $k \in R$.

We are concerned now with finding the Casimir functions of this structure. The defining equation for the Casimir functions, denoted by $C$, is

$$
\Pi^{i j} \partial_{j} C=0 .
$$

The determination of a Casimir in a finite dimensional Hamilton-Poisson system could be done via the algebraic method of Hernández-Bermejo and Fairén (see [3]). Let us observe that the rank of $\Pi$ is constant and equal to 2 . Then, there exist two functionally independent Casimirs associated with our structure. The Casimir function is the solution of the following equations:

$$
x_{3} \frac{\partial C}{\partial x_{2}}+x_{4} \frac{\partial C}{\partial x_{3}}+k \frac{\partial C}{\partial x_{4}}=0, \quad \frac{\partial C}{\partial x_{1}}=0 .
$$


Proposition 1. The functions $C_{1}$ and $C_{2}$ given by

$$
\begin{aligned}
& C_{1}=\frac{1}{2 k}\left(x_{4}^{2}-2 k x_{3}\right), \\
& C_{2}=\frac{1}{3 k}\left(x_{4}^{3}+3 k^{2} x_{2}-3 k x_{3} x_{4}\right), \quad k \in R,
\end{aligned}
$$

are the Casimir functions of the configuration $\Pi$.

Proof. It is easy to see that $\Pi \cdot \nabla C_{1}=0$ and $\Pi \cdot \nabla C_{2}=0$, so the assertion immediately follows.

Remark 2. Remark 4.1 from [2] presents only one Casimir function of the configuration described above. As we can see, the rank of the Poisson configuration matrix equals 2, so there exist two Casimir functions functionally independent. The consequence of this result lies in the analyses of the nonlinear stability of the equilibrium states of the dynamics (1). In addition, we can find two new Hamilton-Poisson structures of the system (1).

Proposition 3. The system (1) admits the following HamiltonPoisson realizations:

$$
\left(R^{4}, \Pi_{1}, C_{1}\right), \quad\left(R^{4}, \Pi_{2}, C_{2}\right)
$$

where

$$
\Pi_{1}=\left[\begin{array}{cccc}
0 & 0 & -x_{2} x_{3}+\frac{1}{k} x_{2} x_{4}^{2} & x_{2} x_{4} \\
0 & 0 & x_{1} x_{3}-\frac{1}{k} x_{1} x_{4}^{2} & -x_{1} x_{4} \\
x_{2} x_{3}-\frac{1}{k} x_{2} x_{4}^{2} & -x_{1} x_{3}+\frac{1}{k} x_{1} x_{4}^{2} & 0 & -k x_{1} \\
-x_{2} x_{4} & x_{1} x_{4} & k x_{1} & 0
\end{array}\right] \text {, }
$$

respectively.

$$
\Pi_{2}=\left[\begin{array}{cccc}
0 & 0 & -\frac{1}{k} x_{2} x_{4} & -x_{2} \\
0 & 0 & \frac{1}{k} x_{1} x_{4} & x_{1} \\
\frac{1}{k} x_{2} x_{4} & -\frac{1}{k} x_{1} x_{4} & 0 & 0 \\
x_{2} & -x_{1} & 0 & 0
\end{array}\right]
$$

The functions $\mathrm{H}$ and $\mathrm{C}_{2}$ are the Casimir functions for the structure $\Pi_{1}$; for the structure $\Pi_{2}$, the Casimir functions are $H$ and $C_{1}$.

Proof. Indeed, we can check that the system (1) can be put into the equivalent form

$$
\begin{gathered}
\dot{x}=\Pi_{1} \cdot \nabla C_{1}=\Pi_{2} \cdot \nabla C_{2}, \\
\Pi_{1} \cdot \nabla H=\Pi_{1} \cdot \nabla C_{2}=\Pi_{2} \cdot \nabla H=\Pi_{2} \cdot \nabla C_{1}=0,
\end{gathered}
$$

as required.

Remark 4. The phase curves of the dynamics (1) are the intersection of the surfaces

$$
H=\text { const., } \quad C_{1}=\text { const., } \quad C_{2}=\text { const. }
$$

\section{Stability Problems}

The equilibrium states of the dynamics (1) are

$$
e_{1}=(0,0, \alpha, \beta), \quad e_{2}=(0, \alpha, 0, \beta), \quad \alpha, \beta \in R .
$$

Using the matrix of the linearized system at the equilibrium of interest, we obtain the following results.
Proposition 5 (see [2]). The equilibrium states $e_{1}$ are spectrally stable for any real values of $\alpha$ and $\beta$.

Proposition 6. The equilibrium states $e_{2}$ are spectrally stable if $\alpha \beta>0$.

Proof. The proof can be obtained immediately using the matrix of the linearized system at the equilibrium of interest and so we will omit any other details.

Now, we are able to study the nonlinear stability of the equilibrium states $e_{1}$ and $e_{2}$.

Proposition 7. The equilibrium states $e_{1}$ are nonlinearly stable for any real values of $\alpha$ and $\beta$.

Proof. We will make the proof using energy-Casimir method [see [4]]. Let

$$
\begin{aligned}
H_{\varphi, \psi} & =H+\varphi\left(C_{1}\right)+\psi\left(C_{2}\right) \\
& =\frac{x_{1}^{2}}{2}+\frac{x_{2}^{2}}{2}+\varphi\left(\frac{x_{4}^{2}}{2 k}-x_{3}\right)+\psi\left(\frac{x_{4}^{3}}{3 k}+k x_{2}-x_{3} x_{4}\right)
\end{aligned}
$$

be the energy-Casimir function, where $\varphi, \psi: R \rightarrow R$ are two smooth real valued functions defined on $R$. Now, the first variation of $H_{\varphi, \psi}$ at the equilibrium $e_{1}$ equals zero if

$$
\dot{\varphi}\left(\frac{\beta^{2}}{2 k}-\alpha\right)=0, \quad \dot{\psi}\left(\frac{\beta^{3}}{3 k}-\alpha \beta\right)=0 .
$$


If we choose now the functions $\varphi$ and $\psi$ such that

$$
\ddot{\varphi}\left(\frac{\beta^{2}}{2 k}-\alpha\right)>0, \quad \ddot{\psi}\left(\frac{\beta^{3}}{3 k}-\alpha \beta\right)>0,
$$

the second variation of $H_{\varphi, \psi}$ at the equilibrium $e_{1}$ is positively defined, so we can conclude that the equilibrium points $e_{1}$ are nonlinearly stable.

Proposition 8. If $\alpha, \beta<0$, the equilibrium states $e_{2}$ are nonlinearly stable.

Proof. To obtain the nonlinear stability result, for this time, we consider the function

$$
\begin{aligned}
H_{\varphi, \psi}= & k C_{2}+\varphi(H)+\psi\left(k C_{1}\right) \\
= & \frac{x_{4}^{3}}{3}+k^{2} x_{2}-k x_{3} x_{4} \\
& +\varphi\left(\frac{x_{1}^{2}}{2}+\frac{x_{2}^{2}}{2}\right)+\psi\left(\frac{x_{4}^{2}}{2}-k x_{3}\right)
\end{aligned}
$$

$$
L=\left[\begin{array}{cccccc}
0 & x_{2}-2 x_{1} & 2 x_{2}+x_{1} & 0 & 0 & 0 \\
-x_{2}+2 x_{1} & 0 & -2 i & 0 & 0 & 0 \\
-2 x_{2}-x_{1} & 2 i & 0 & 0 & 0 & 0 \\
0 & 0 & 0 & 0 & i x_{3}+\frac{i}{\sqrt{2}} x_{4}-i \sqrt{2} & p \\
0 & 0 & 0 & -i x_{3}-\frac{i}{\sqrt{2}} x_{4}+i \sqrt{2} & 0 & q \\
0 & 0 & 0 & -p & -q & 0
\end{array}\right]
$$

$$
\begin{aligned}
& \dot{\varphi}\left(\frac{\alpha^{2}}{2}\right)=-\frac{k^{2}}{\alpha}, \quad \dot{\psi}\left(\frac{\beta^{2}}{2}\right)=-\beta, \\
& \ddot{\varphi}\left(\frac{\alpha^{2}}{2}\right)>0, \quad \ddot{\psi}\left(\frac{\beta^{2}}{2}\right)>-\frac{1}{\beta} .
\end{aligned}
$$

Using the same energy-Casimir method, we can conclude that the equilibrium state $e_{2}$ is nonlinearly stable if $\alpha, \beta<$ 0 .

\section{Lax Formulation}

The dynamics (1) allow a Lax formulation:

$$
\dot{L}=[L, B]
$$

where

where $p=1-(1 / \sqrt{2}) x_{3}-x_{4}, q=-1+(1 / \sqrt{2}) x_{3}-(k / \sqrt{2})$, and

$$
B=\left[\begin{array}{cccccc}
0 & -\frac{1}{2} x_{2}+x_{1} & -x_{2}-\frac{1}{2} x_{1} & 0 & 0 & 0 \\
\frac{1}{2} x_{2}-x_{1} & 0 & i+x_{3} & 0 & 0 & 0 \\
x_{2}+\frac{1}{2} x_{1} & -i-x_{3} & 0 & 0 & 0 & 0 \\
0 & 0 & 0 & 0 & \sqrt{2} x_{1} & i x_{1} \\
0 & 0 & 0 & -\sqrt{2} x_{1} & 0 & -i x_{1} \\
0 & 0 & 0 & -i x_{1} & i x_{1} & 0
\end{array}\right]
$$

\section{Numerical Integration}

We will discuss now the numerical integration of (1) via the Lie-Trotter formula [see [5]] and Kahan's integrator [see [6]]. A numerical comparison of the two results is presented in Figures 1 and 2. Now, splitting the Hamiltonian vector field $X_{H}$ as

$$
X_{H}=X_{H_{1}}+X_{H_{2}}
$$

where $H_{1}=(1 / 2) x_{1}^{2}$ and $H_{2}=(1 / 2) x_{2}^{2}$; the integral curves of the vector fields $X_{H_{1}}$ and $X_{H_{2}}$ are given by

$$
\begin{aligned}
& c_{1}\left(t, x_{1}(0), x_{2}(0), x_{3}(0), x_{4}(0)\right) \\
& \quad=A(t)\left[\begin{array}{llll}
x_{1}(0) & x_{2}(0) & x_{3}(0) & x_{4}(0)
\end{array}\right], \\
& c_{2}\left(t, x_{1}(0), x_{2}(0), x_{3}(0), x_{4}(0)\right) \\
& \quad=B(t)\left[\begin{array}{llll}
x_{1}(0) & x_{2}(0) & x_{3}(0) & x_{4}(0)
\end{array}\right],
\end{aligned}
$$




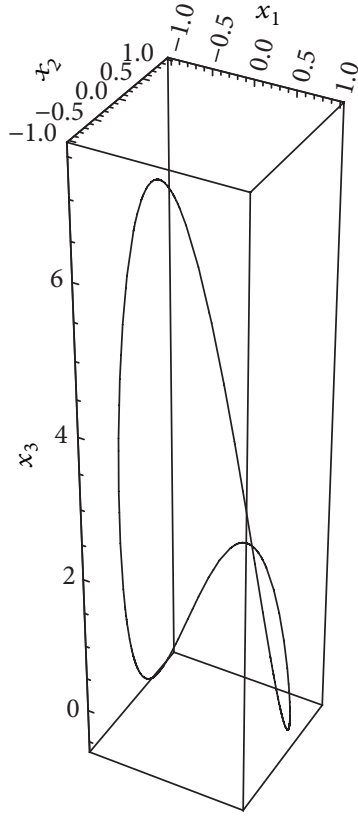

Figure 1: The phase curves of the system (1), projection on $\left(O x_{1} x_{2} x_{3}\right)$ plane $(k=1)$.

where

$$
\begin{aligned}
& A(t)=\left[\begin{array}{cccc}
1 & 0 & 0 & 0 \\
-\frac{k\left[x_{1}(0)\right]^{2} t^{3}}{6} & 1 & -x_{1}(0) t & \frac{\left[x_{1}(0)\right]^{2} t^{2}}{2} \\
\frac{k x_{1}(0) t^{2}}{2} & 0 & 1 & -x_{1}(0) t \\
-k t & 0 & 0 & 1
\end{array}\right], \\
& B(t)=\left[\begin{array}{cccc}
1 & 0 & x_{2}(0) t & 0 \\
0 & 1 & 0 & 0 \\
0 & 0 & 1 & 0 \\
0 & 0 & 0 & 1
\end{array}\right] .
\end{aligned}
$$

Now, the Lie-Trotter integrator can be written as

$$
\left[\begin{array}{llll}
x_{1}^{k+1} & x_{2}^{k+1} & x_{3}^{k+1} & x_{4}^{k+1}
\end{array}\right]^{t}=A(t) B(t)\left[\begin{array}{llll}
x_{1}^{k} & x_{2}^{k} & x_{3}^{k} & x_{4}^{k}
\end{array}\right]^{t}
$$

or, explicitly, as

$$
\begin{aligned}
x_{1}^{k+1}= & x_{1}^{k}+x_{2}[0] t x_{3}^{k}, \\
x_{2}^{k+1}= & -\frac{k\left[x_{1}(0)\right]^{2} t^{3}}{6} x_{1}^{k} \\
& +x_{2}^{k}-\left[x_{1}(0) t+\frac{k\left[x_{1}(0)\right]^{2} x_{2}(0) t^{4}}{6}\right] x_{3}^{k} \\
& +\frac{\left[x_{1}(0)\right]^{2} t^{2}}{2} x_{4}^{k},
\end{aligned}
$$

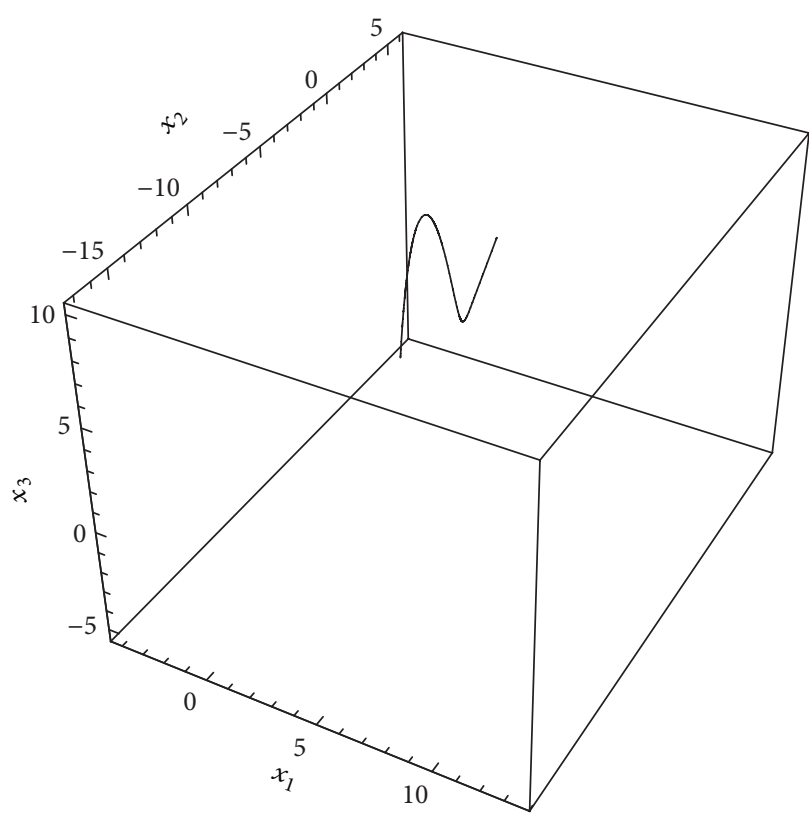

FIGURE 2: The Lie-Trotter integrator of the system (1), projection on $\left(O x_{1} x_{2} x_{3}\right)$ plane $\left(k=1, x_{1}(0)=x_{2}(0)=x_{3}(0)=1\right)$.

$$
\begin{aligned}
x_{3}^{k+1}= & \frac{k x_{1}(0) t^{2}}{2} x_{1}^{k} \\
& +\left[\frac{k x_{1}(0) x_{2}(0) t^{3}}{2}+1\right] x_{3}^{k}-x_{1}(0) t x_{4}^{k}, \\
x_{4}^{k+1}= & -k t x_{1}^{k}-k x_{2}(0) t^{2} x_{3}^{k}+x_{4}^{k} .
\end{aligned}
$$

Some of its properties are sketched in the following proposition.

Proposition 9. The numerical integrator (24) preserves the Poisson structures $\Pi, \Pi_{1}$, and $\Pi_{2}$; it preserves the Casimirs $C_{1}$ and $C_{2}$, but it does not preserve the Hamiltonian $H$ of the configuration $\left(R^{3}, \Pi\right)$. In addition, its restriction to the coadjoint orbits

$$
x_{3}^{2}-2 k x_{2}=\text { const., } \quad x_{4}^{3}+3 k^{2} x_{2}-3 k x_{3} x_{4}=\text { const } .
$$

gives rise to a symplectic integrator.

Proof. The numerical integrator (24) preserves the Poisson structures and the Casimirs of the above configuration because $c_{1}$ and $c_{2}$ are flows of some Hamiltonian vector fields; hence they are Poisson ones. For the same reasons, the restriction to the coadjoint orbits gives rise to a symplectic integrator. The numerical integrator (24) does not preserve the Hamiltonian because

$$
\left\{H_{1}, H_{2}\right\} \neq 0 \text {. }
$$


The numerical simulation of the Lie-Trotter integrator, using Mathematica 8.0, is presented in Figure 2.

Let us observe now that Kahan's integrator, associated with the dynamics (1), has the following form:

$$
\begin{aligned}
& x_{1}^{k+1}-x_{1}^{k}=\frac{h}{2}\left(x_{2}^{k} x_{3}^{k+1}+x_{3}^{k} x_{2}^{k+1}\right), \\
& x_{2}^{k+1}-x_{2}^{k}=-\frac{h}{2}\left(x_{1}^{k} x_{3}^{k+1}+x_{3}^{k} x_{1}^{k+1}\right), \\
& x_{3}^{k+1}-x_{3}^{k}=-\frac{h}{2}\left(x_{1}^{k} x_{4}^{k+1}+x_{4}^{k} x_{1}^{k+1}\right), \\
& x_{4}^{k+1}-x_{4}^{k}=-h k\left(x_{1}^{k}+x_{1}^{k+1}\right) .
\end{aligned}
$$

After a log but straightforward computation, we are leading to the following properties of Kahan's integrator.

Proposition 10. Kahan's integrator (27) has the following properties: it does not preserve the Poisson structures $\Pi, \Pi_{1}$, $\Pi_{2}$, it does not preserve the Casimirs $C_{1}, C_{2}$, and it does not preserve Hamiltonian $\mathrm{H}$.

Figure 3 represents the numerical simulation of the Kahan integrator.

Remark 11. Using Mathematica 8 we obtained numerical simulations for a Poisson integrator (the Lie-Trotter one) and a non-Poisson integrator (Kahan one). As we can see, the Lie-Trotter integrator gives us a better approximation of the trajectory movement, but both of them are quite different from Figure 1, which is the exact solution of the system (1).

\section{Conclusion}

A lot of mechanical problems, like the cinematic model of an automobile with $(n-3)$ trailers [7], the underwater vehicle dynamics [1], the spacecraft dynamics [8], the molecular motion in the context of coherent control of quantum dynamics [9], and the ball-plate problem [10] or the control tower problem from air traffic [11] or the Lagrange system [12] have been modeled as a set of differential equations with the configuration space on a matrix Lie group. Finding a Hamilton-Poisson formulation for these systems is an important first step. The Hamilton-Poisson formulation allows us to study the systems form mechanical geometry points of view by giving the specific tools to study the nonlinear (Lyapunov) stability of the equilibrium states (by using energy-Casimir method), the existence of the periodic orbits (by using the Lyapunov center theorem), the bifurcation phenomena, the numerical integration (by using Poisson integrators, like Lie-Trotter one), integrability, and so forth. For such a formulation, finding the Casimir functions is an important step: they could provide other Poisson structures and they play a major role in the study of the nonlinear stability with all the known energy methods.

In addition, the Hamilton-Poisson realization offers us the possibility to find the exact solution of the system as the intersection of some surfaces, the surfaces equation

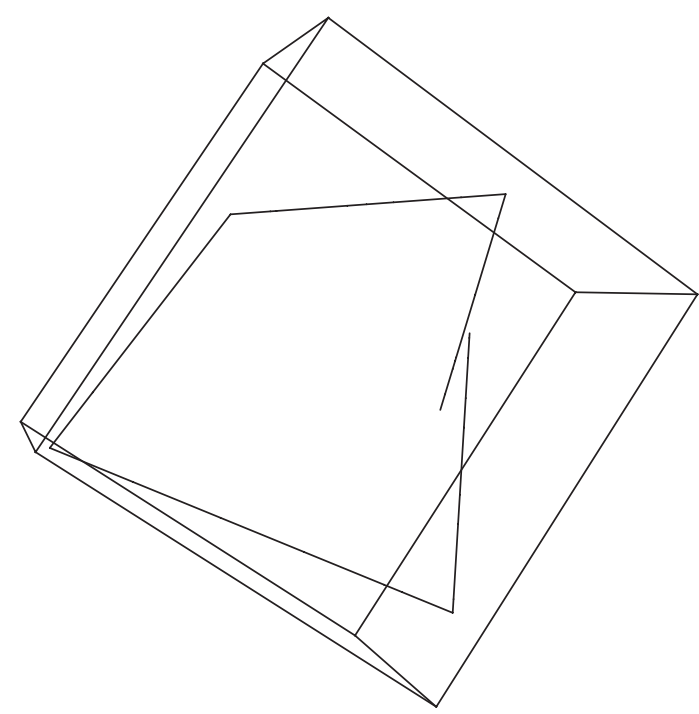

FIgURE 3: The Kahan integrator of the system (1), projection on $\left(O x_{1} x_{2} x_{3}\right)$ plane $\left(h=1, k=1, x_{1}(0)=x_{2}(0)=x_{3}(0)=1\right)$.

being given by the Hamiltonian and the Casimirs of our configuration.

Studying the system from the Poisson geometry point of view may offer us the opportunity to find the connection between the dynamical properties of the system and the geometry of the image of a vector valued constant of motion (the energy-Casimir mapping, in our case) and can help us to detect as many as possible dynamical elements (e.g., equilibria, periodic orbits, and homoclinic and heteroclinic connections) and dynamical behavior (e.g., stability, bifurcation phenomena for equilibria, periodic orbits, and homoclinic and heteroclinic connections) by just looking at the image of this mapping (see [13]).

\section{Conflict of Interests}

The author declares that there is no conflict of interests regarding the publication of this paper.

\section{Acknowledgment}

The author would like to thank the referees very much for their valuable comments and suggestions.

\section{References}

[1] N. E. Leonard, Averaging and motion control of systems on Lie groups [Ph.D. thesis], University of Maryland, 1994.

[2] M. Craioveanu, C. Pop, and M. Puta, "Geometrical aspects in the dynamics of an automobil with two trailers, differential geometry and applications," in Proceedings of the Satellite Conference of ICM in Berlin, pp. 347-353, Brno, Czech Republic, August 1998.

[3] B. Hernández-Bermejo and V. Fairén, "Simple evaluation of Casimir invariants in finite-dimensional Poisson systems," Physics Letters A, vol. 241, no. 3, pp. 148-154, 1998. 
[4] M. Puta, Hamiltonian Mechanical Systems and Geometric Quantization, Kluwer Academic Publishers, Dordrecht, The Netherlands, 1993.

[5] M. Puta, "Lie-Trotter formula and Poisson dynamics," International Journal of Bifurcation and Chaos in Applied Sciences and Engineering, vol. 9, no. 3, pp. 555-559, 1999.

[6] W. Kahan, "Unconventional numerical methods for trajectory calculation," Unpublished Lecture Notes, 1993.

[7] M. Puta and C. Pop, "Control and stability in the kinematic model of an automobile with $n-3$ trailers," Reports on Mathematical Physics, vol. 45, no. 1, pp. 147-154, 2000.

[8] M. Puta, "Stability and control in spacecraft dynamics," Journal of Lie Theory, vol. 7, no. 2, pp. 269-278, 1997.

[9] M. Dahleh, A. Peirce, H. A. Rabitz, and V. Ramakrishna, "Control of molecular motion," Proceedings of the IEEE, vol. 84, no. 1, pp. 7-13, 1996.

[10] C. Pop, A. Aron, and C. Petrişor, "Geometrical aspects of the ball-plate problem," Balkan Journal of Geometry and its Applications, vol. 16, no. 2, pp. 114-121, 2011.

[11] S. S. Sastry, R. Montgomery, and G. Walsh, "Optimal path planning on matrix lie groups," in Proceedings of the 33rd IEEE Conference on Decision and Control, pp. 1258-1263, 1995.

[12] C. Dăniasă, C. Hedrea, C. Pop, and M. Puta, "Some geometrical aspects in the theory of Lagrange system," The Tensor Society. Tensor. New Series, vol. 69, pp. 83-87, 2008.

[13] C. Lăzureanu and T. Bînzar, "On a Hamiltonian version of controls dynamic for a drift-free left invariant control system on $G_{4}$," International Journal of Geometric Methods in Modern Physics, vol. 9, no. 8, Article ID 1250065, 2012. 


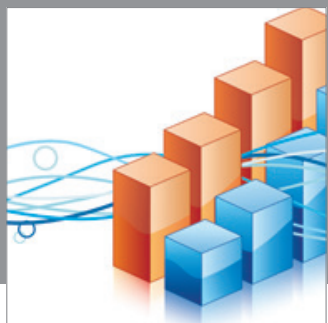

Advances in

Operations Research

mansans

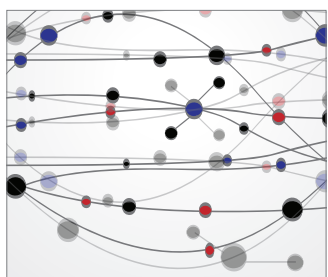

The Scientific World Journal
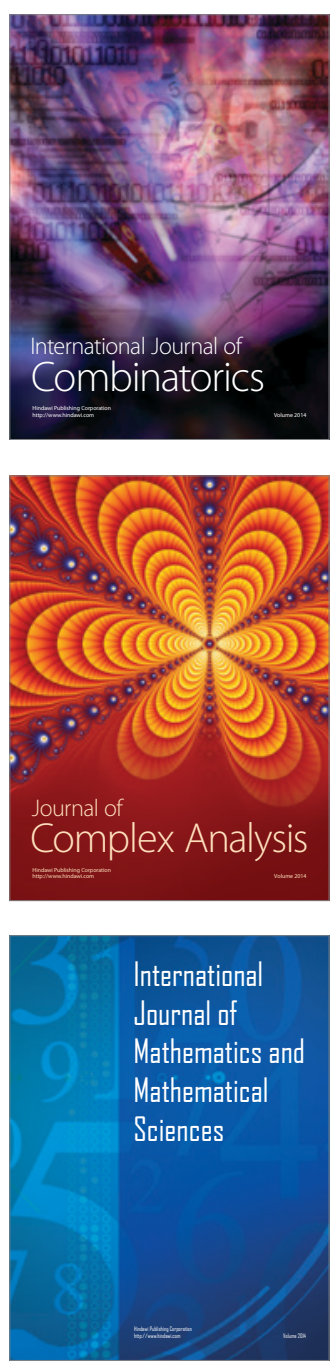
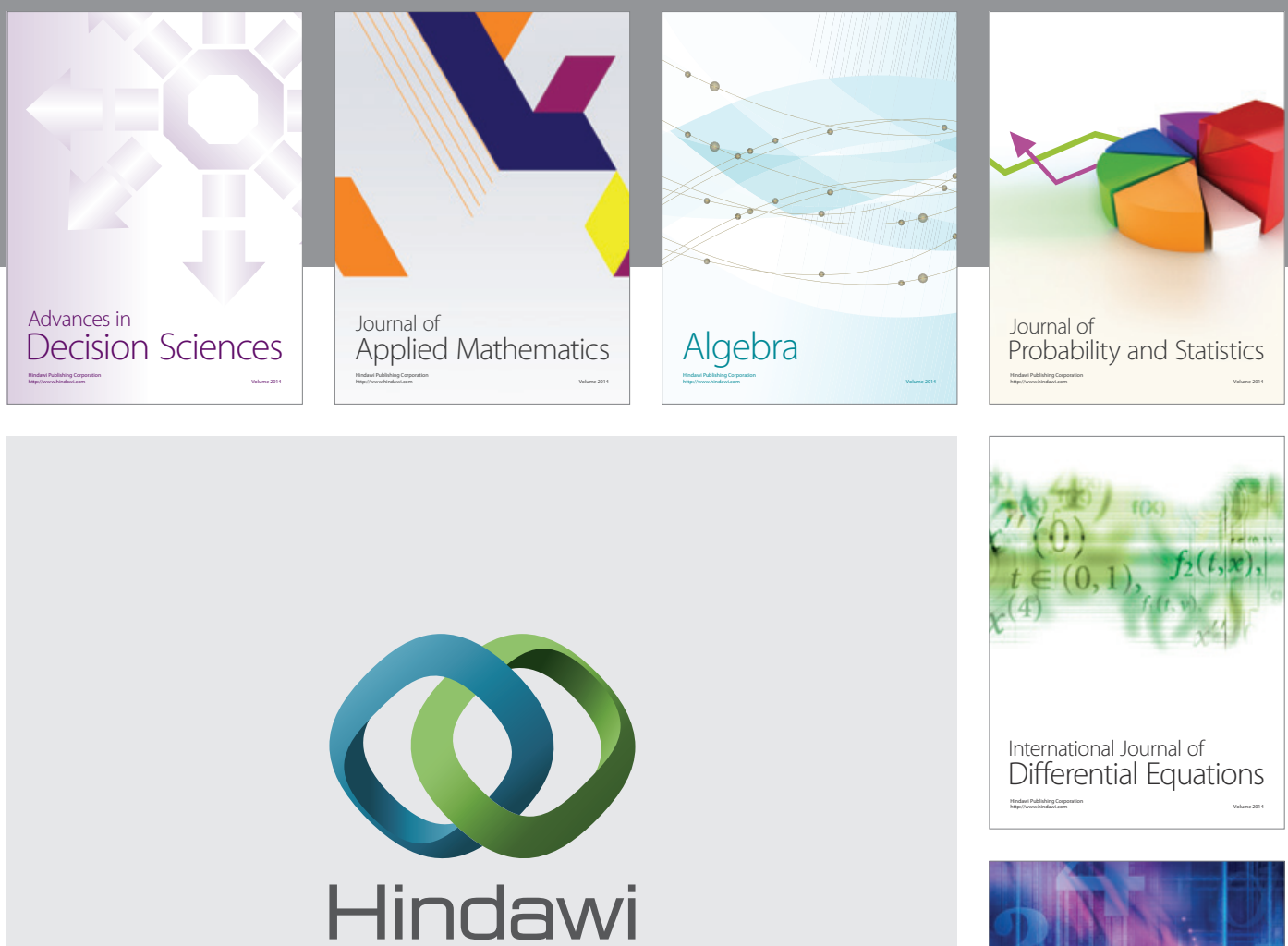

Submit your manuscripts at http://www.hindawi.com
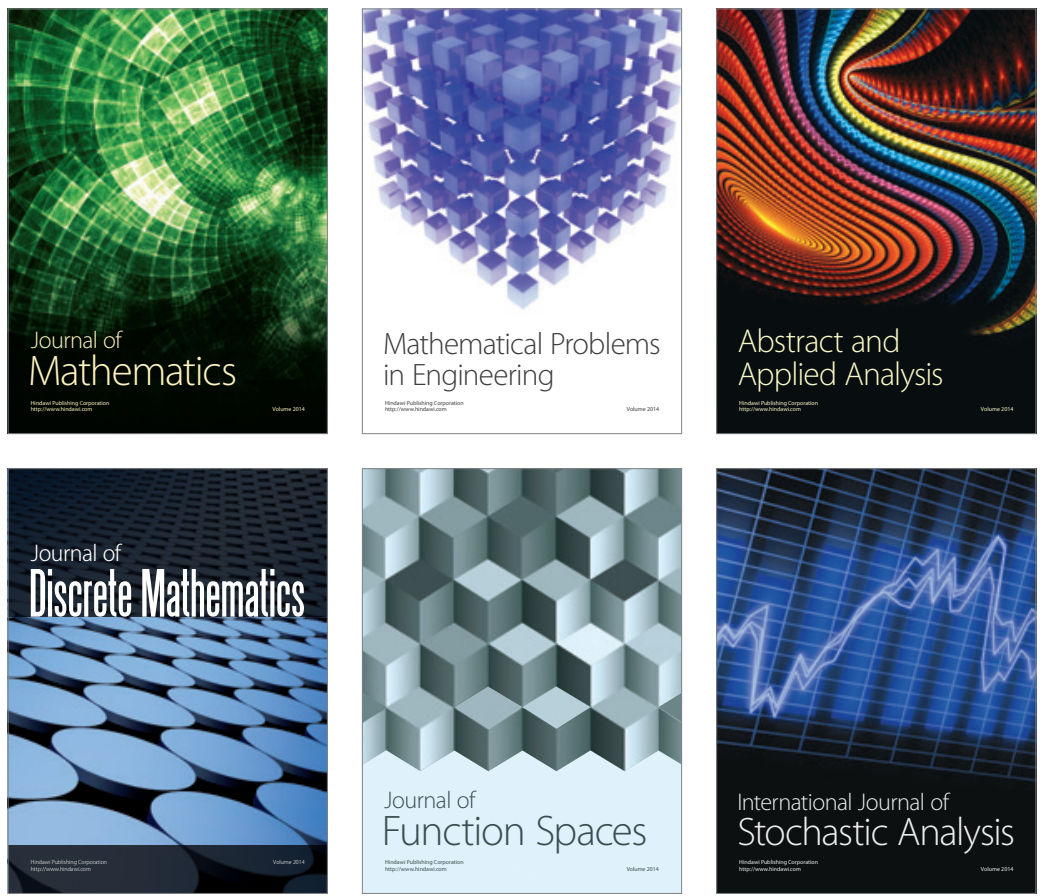

Journal of

Function Spaces

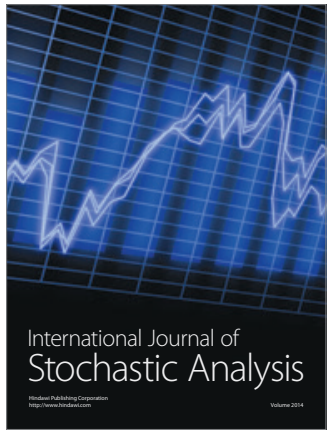

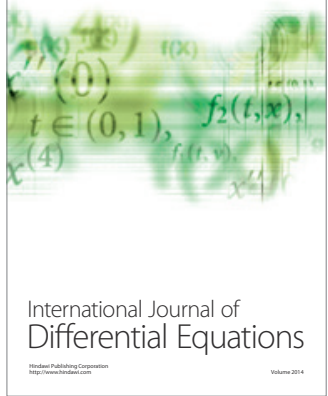
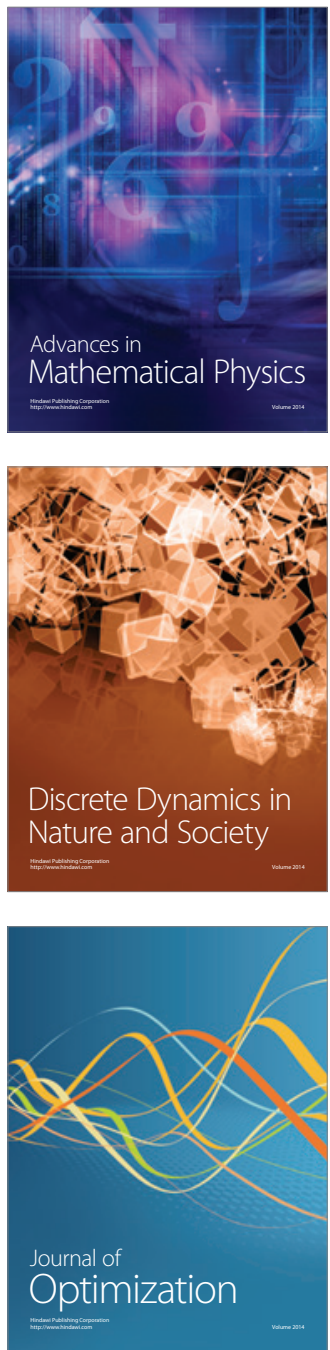